\title{
Modeling of QoS in the Industrial Ethernet Switches
}

\author{
E. Kizilov ${ }^{1}$, N. Konnov ${ }^{1}$, D. Pashchenko ${ }^{1}$ and D. Trokoz ${ }^{1}$ \\ ${ }^{1}$ Department of Computer, Penza State University, Penza, 440012, Russia
}

Keywords: Petri nets, industrial Ethernet, quality of service.

\begin{abstract}
The principles of the simulation of industrial Ethernet switch with the support of the quality of service hierarchical colored time Petri nets by means of a CPN Tools package are stated. The research example by means of the offered model of a diverse traffic jitter for servicing of which a combination of algorithms of strict and Round Robin prioritization was applied in the switch is given.
\end{abstract}

\section{Introduction}

In modern telecommunications infrastructure Automated process control systems technology is widely used dial-Ethernet $[1,2]$, which is facilitated by its qualities, such as:

- $\quad$ high-speed of transmission, that provides compliance with hard real-time;

- $\quad$ easy integration with Internet / Intranet, including application layer protocol;

- $\quad$ the possibility for organization of a variety of networking topologies;

- The emergence of low-cost switches, decided to issue a non-deterministic Ethernet.

The main requirement for equipment of Industrial Ethernet is the support of the quality of service (QoS), that is capable of supporting the requirements of the delay and its variation (jitter) while transmitting real-time traffic. Mechanisms QoS (marking and traffic classification, queuing, congestion control, bandwidth management) provide guaranteed bandwidth allocation and prioritization of separate application traffic [3].

Selection of optimal settings of switches QoS is a complex task that can be projected network simulation modeling, for which the authors are encouraged to use the device of color, hierarchical, temporal Petri nets that is implemented in the package CPN Tools [4].

\section{Discussed Problems}

In developing of the switch model the top-down design methodology was used. Model quad-switch on the top level of the hierarchy includes (Fig. 1):

- subnet «InPort» and «OutPort», which simulate the switch ports that provide access to communication lines in full duplex mode;

- subnet «Switch», modeling promotion frame from the input port to a specific output port in accordance with the switching table (supports both static and dynamic modes of reference tables);

- subnet «Buf» which simulate frame buffer directed to the appropriate output port.

For modeling of interaction protocol of network devices is very important to use the color marker device effectively.

Network traffic is modeled by a sequence of tokens received at the input ports and outputed from the output ports, colors of which form a tuple frm fields corresponding to the frame format Ethernet $802.1 \mathrm{q} / \mathrm{p}$ [6]: source and destination addresses, the field tag with information about belonging to a specific priority level, a field indicating the length of the payload of the frame, as well as service time field the parish and the outcome of the frame (used to collect statistics). The variety of colors frame is also used, it is the bearer, which may be either busy sending a frame frm, or free avail, that allows to detect and handle events related to the presence or absence of the frame. Additionally, the tokens are introduced, they reflect the color characteristics of the utility model (queue size, amount of data transferred, the queue attribute cleaning et al.). To describe the colors, functions and variables language CPN ML is used [4]. 
Realization of subnets «InPort», «OutPort» and «Switch» is given in [5] Therefore, the authors focused on the modeling of mechanisms QoS, which are implemented in the subnet «Buf», specially of the introduction of classes of service for different types of traffic, congestion control support of different algorithms of queue management.

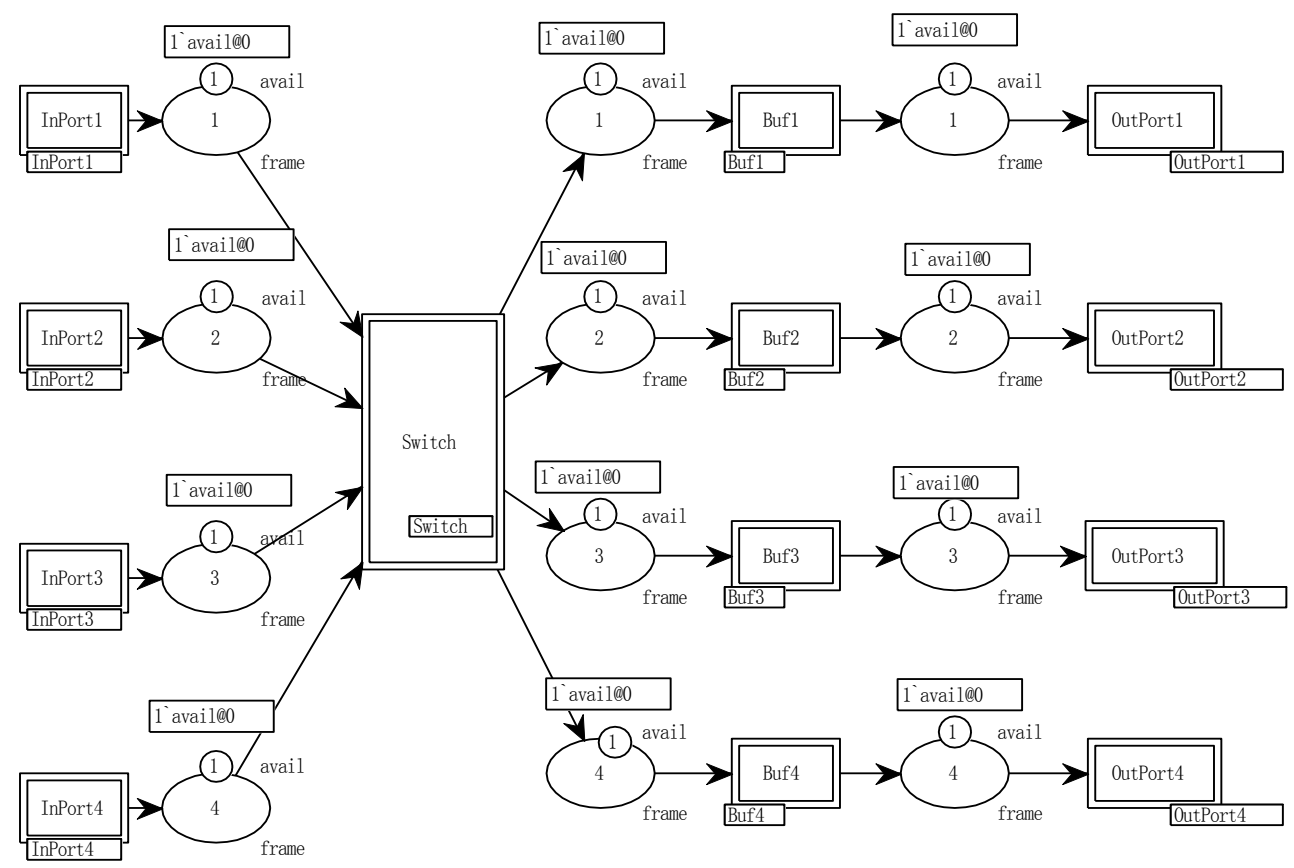

Fig. 1. General model the four-port quad-switch.

In Ethernet switches L2 applied strictly priority (SP) queuing algorithm, in which the queues are serviced in accordance with strictly defined priority and Round Robin (RR) algorithms that maintain consistent service queues, ensuring access to each output port. Usually RR algorithm is implemented in versions allowing to regulate the bandwidth or each channel:

- Weighted Round Robin (WRR) - specifies the weight of the number of frames sent from the queue in a single session service, but allocated bandwidth depends on the length of the transmitted packets;

- Deficit Round Robin (DRR) - allows you to accurately allocate the band that is regardless of the length of the transmitted packets.

Frequently combined options of queuing SP + WRR or SP + DRR, are used, they allow to ensure critical traffic minimum delay [7].

Network «Buf», which implements SP + WRR, is shown in Fig. 2 and includes the following components:

1. «Buffer in» and «Buffer out» - input and output ports of the buffer output port.

2. «Classifier» - incoming traffic classifier that works in terms of priority field token color frm the next frame. and sending it to one of four queues for processing.

3. «Queue»- queue of a given size FIFO, containing tokens frames awaiting processing. In subnet, the count of the current queue size, displayed in the token position Size Queue, is also made, and reset of the tokens of overflow queue is carried out, as well. Reading from the queue is allowed in the position token Start selection. Implementation of Queue is discussed in details in [8].

4. «Scan»- subnet, which implements queuing SP + WRR. Strict priority queuing algorithm SP 1 is implemented by predicate formation tokens in positions Start selection1 subnet Scan (Fig. 3), cyclic 2-4 queuing algorithm is given by transition Scan, which is triggered every time a frame queues have tokens (token color positions Size Queue 1 is the color token at one of the remaining positions Size Queue nonzero) and the output port of the switch is free (in the position of the token has Outport free). When triggered, the transition to a 
position Start selection2 - Start selection4, to be determined as appropriate predicates that take into account the color of the token in position $\mathbf{N}$, which points to the next "candidate" for maintenance and modified by passing through the frame tokens subnet "Band" at the output port.

5. «Bend» subnet realizing account of balance to set bandwidth of each queue. WRR algorithm for position Max Size Queue contains a token whose color sets the weighting factor - the maximum number of shots that can be taken to the appropriate queue in a single session service. Position $\mathbf{K}$ contains a token, the color of which is used to control the amount transferred per session of service tokens of frames. When transferring the last of the possible frames in the session triggering transition Band modifies the color of token in position $\mathbf{N}$, translating a pointer to the next queue (Fig. 4).

6. Network «Port»,to say briefly, is not a part of the «Buf» and is given to illustrate the formation of serialization delay in the transmission of frames of the queue, the duration of which depends on the size with the preamble and frame interval.

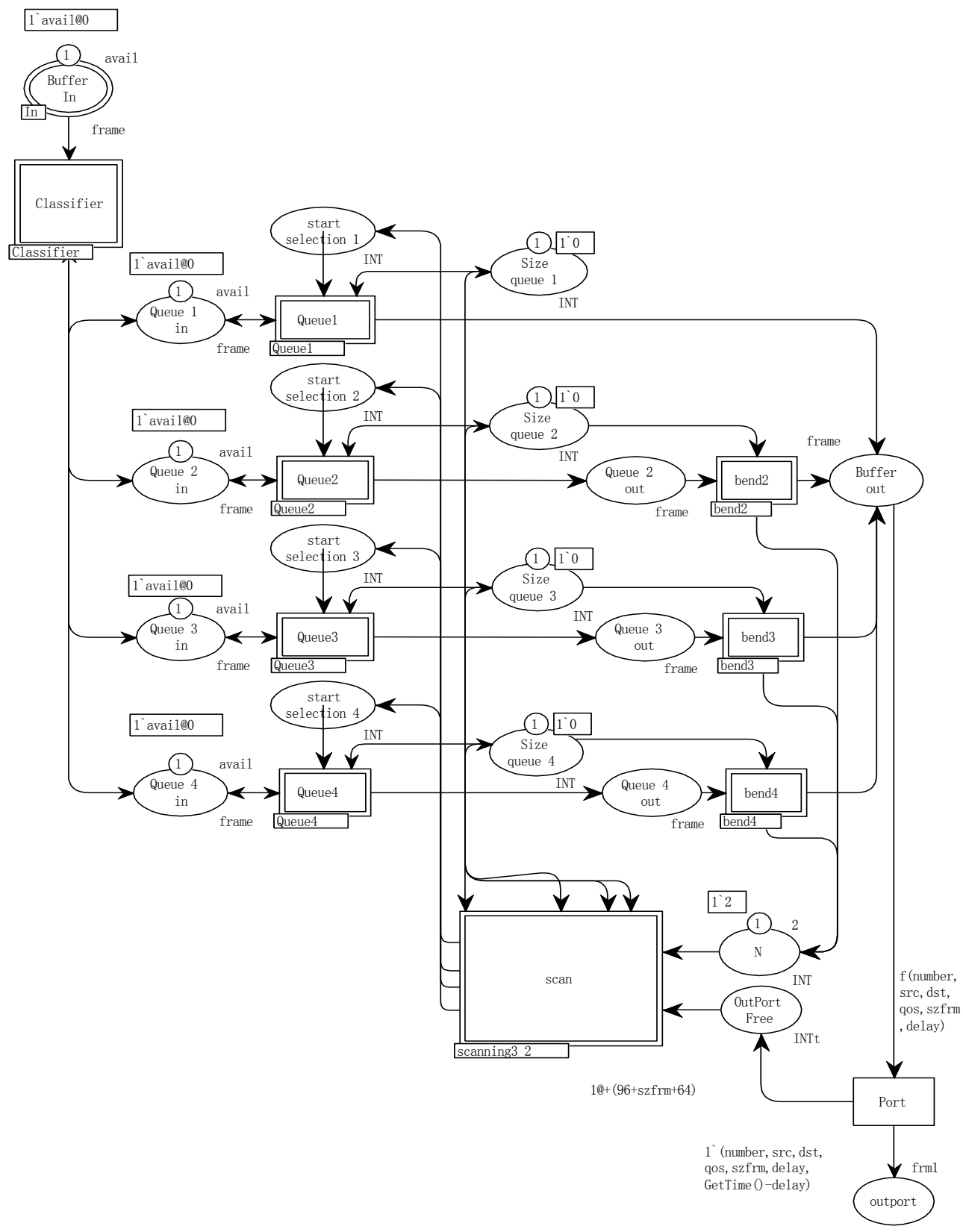

Fig. 2. Subnet of the Buffer of Output Port. 


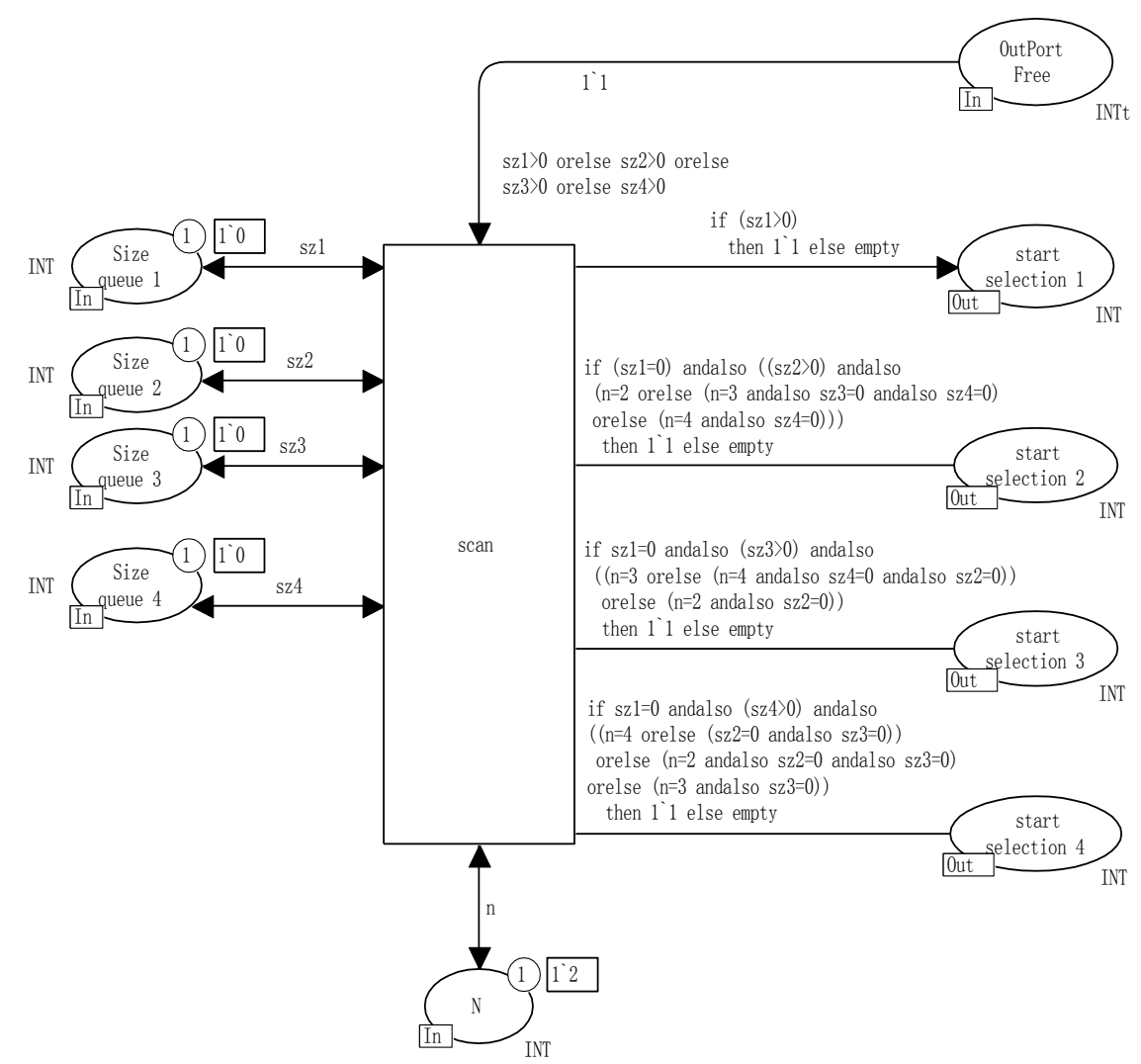

Fig. 3. Subnet of service (scanning) of queues.

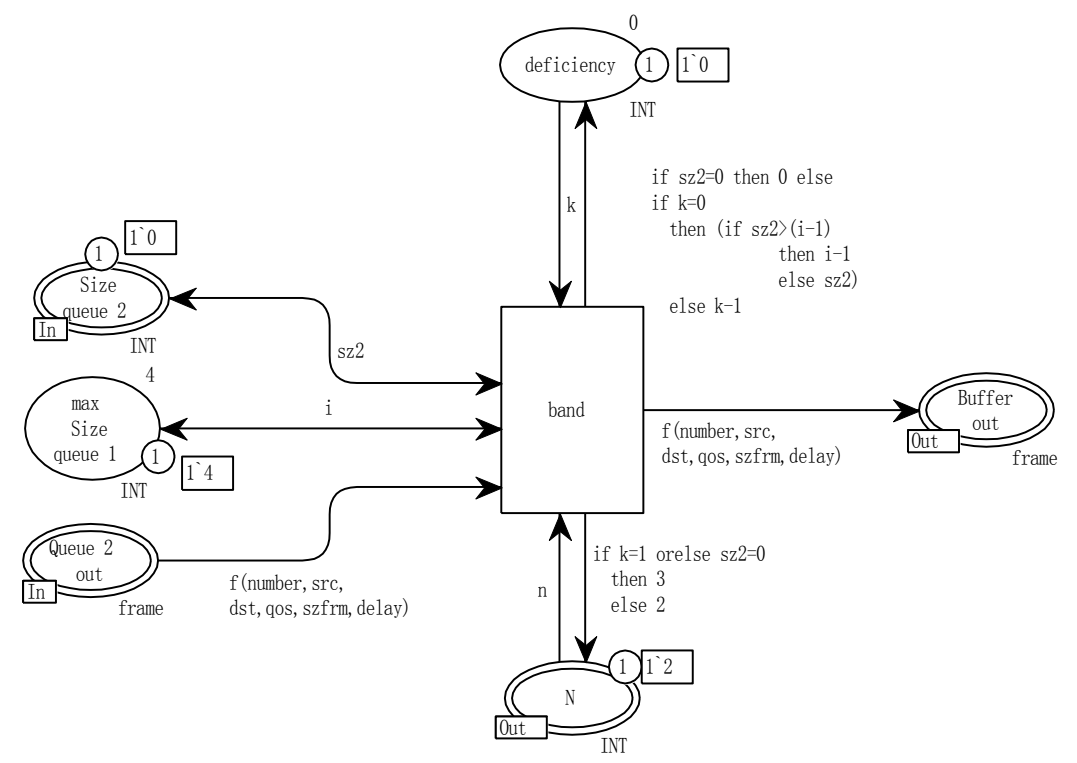

Fig. 4. A subnet of regulation of a strip for algorithm of WRR.

As an example of the proposed model, below the evaluation results of the distribution function of jitter frames with different classes of QoS are shown (Fig. 5).

Model time is expressed in clock intervals, which are equal to $100 \mathrm{~ns}, 10 \mathrm{~ns}$, or Ethernet for Fast Ethernet). The input traffic is modeled by using a special Petri nets [9]. It is a stream of frames belonging equally to 4 QoS classes, and going through the following time intervals exponentially distributed with a average value of the delivery period 12000. Random length of a frame has the following distribution: $25 \%$ of all the frames are the minimum and maximum length (512 and 12176, respectively), the remaining length is evenly distributed in the remaining range. The load factor of the channel is 0.798 . An algorithm for servicing the traffic class is set 1 SP,for classes 2,3,4 WRR, with a division of bandwidth as 4: 4: 2 . 


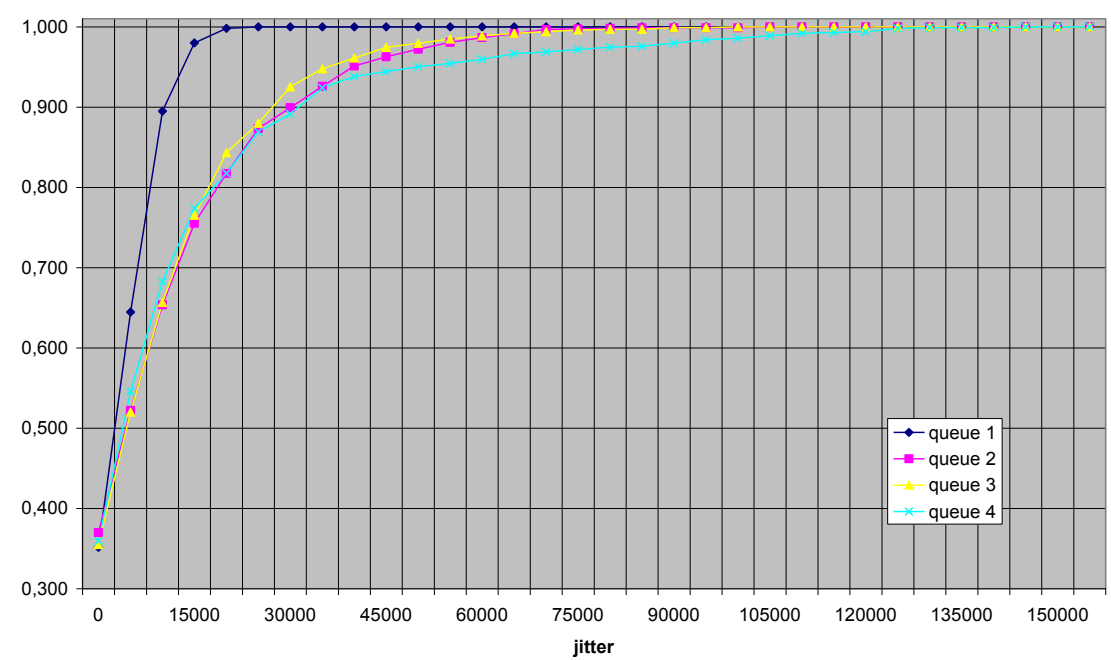

Fig. 5. Integrated function of distribution jitter frames with different QoS classes.

The average and maximum jitter of different classes of traffic are listed in Table 1 below, from that it can be seen that the average jitter value in the high-priority traffic is 2.5-3 times, and the maximum value in the 5-7 times lower than that of traffic served by a cyclic priority.

Table 1. Average and Maximum Jitter

\begin{tabular}{|c|c|c|}
\hline Class of traffic & Average jitter & Maximum jitter \\
\hline 1 & 3917 & 20578 \\
\hline 2 & 10121 & 102323 \\
\hline 3 & 9518 & 97676 \\
\hline 4 & 11372 & 143193 \\
\hline
\end{tabular}

Long "tail" of the distribution of traffic jitter 4, says that the band set to it does not match its real share in the general flow of frames.

\section{Conclusions}

The proposed model allows to investigate the probabilistic and temporal characteristics (average delay, jitter, packet loss share) of heterogeneous traffic, dial-in Industrial Ethernet networks with support for QoS.

\section{Acknowledgements}

This work is made in the framework of the Federal Targeted Programme "Research and development in priority areas of elaboration of the science and technology complex of Russia for 2014-2020” (agreement of 06.19.2014 № 14.574.21.0045)

\section{References}

[1] Olifer N., OliferV. Computer Networks: Principles, Technologies and Protocols for Network Design, Wiley E-Text, 2007, p 1000

[2] Grübmeyerand R., Rupp S. Ethernet-based fieldbuses for industrial networks: the basics Industrial Ethernet Book, http://www.iebmedia.com/index.php?id= $8249 \&$ parentid $=63 \&$ themeid $=255 \& \mathrm{hft}=66 \&$ showdetail $=$ true $\& b b=1$

[3] Hirschmann Network Systems. White Paper. Real Time Services (QoS) In Ethernet Based Industrial Automation Networks. http://www.ictglobal.com/whitepapers/QoS.pdf

[4] Jensen K., Kristensen L. Coloured Petri Nets: modelling and validation of concurrent systems. - Springer-Verlag, 2009 - p. 384. 
[5] Zaitsev D.A. Switched LAN simulation by colored Petri nets // Mathematics and Computers in Simulation, vol. 65, № 3, 2004,3. pp. 245-249.

[6] Ek Niclas (1999) Department of Electrical Engineering, Helsinki University of Technology. $\begin{array}{lllllll}\text { IEEE } & 802.1 & \text { P,Q } & - & \text { QoS } & \text { on }\end{array}$ http://www.tml.tkk.fi/Opinnot/Tik110.551/1999/papers/08IEEE802.1QosInMAC/qos.html

[7] Vegesna, S.: IP Quality of Service. Cisco Press, 2001, 359p.

[8] Mehanov V.B., Kizilov E.A. Modelirovanie tsvetnyimi setyami Petri obsluzhivaniya ocheredey algoritmom WRR // Trudyi IX Mezhdunarodnoy nauchno-tehnicheskoy konferentsii "Novyie informatsionnyie tehnologii i sistemyi", Ch. 1. Penza: Izd-vo PGU, 2010, s. 67-73.

[9] Nikishin K.I., Konnov N.N., Domnin A.L. Modelirovanie trafika seti Ethernet tsvetnyimi setyami Petri. Sb. materialov I Mezhdunar. nauch.-prakt. konf «Sovremennyie problemyi kompyuternyih nauk (SPKN-2013)» Penza : Izd-vo PGU, 2013, s. 120-123. 